\title{
Uncooled Amorphous Silicon IRFPA for High Performance and High Volume Applications
}

\author{
J.L. Tissot, A. Crastes, M. Vilain, O. Legras, S. Tinnes, C. Minassian, P. Robert, B. Fieque \\ ULIS - ZI les Iles Cordées, BP27 - 38113 Veurey Voroize, France
}

\begin{abstract}
For more than 10 years now, uncooled sensors have given new opportunities in the IR field of applications by being able to be produce in large volume. Compared to cooled technology, uncooled detectors offer many interesting advantages: high reliability, lower cost ... whereas the performance is high enough for a lot of applications. Thermography, building inspection, enhanced driver vision and military (thermal weapon sight, low altitude UAV sensor) are applications which can be provided with affordable IR focal plane arrays....

As uncooled IR sensors are mainly dedicated to these high volume applications, any uncooled IRFPA technology has to be able to provide high performance sensors but also to be producible in large volume at a minimum cost.

The high level of accumulated expertise by ULIS and CEA/LETI on uncooled microbolometers made from amorphous silicon layer enables ULIS to develop a full range of IRFPA formats from $160 \times 120$ to $1024 \times$ 768 pixels with $25 \mu \mathrm{m}$ and $17 \mu \mathrm{m}$ pixel-pitch, designed for high end and high volume applications.

The detector ROIC designs rely on a simple architecture (detector configuration addressed by a serial link for user defined amplifier gain, windowing capability ...) which enables easier systems upgrade and therefore a reduced system development non recurrent cost.

The packaging technique depends on the application environment and the production volume in order to fit with the market expectation. Starting from metallic and ceramics package, very advanced new technique is under development in order to reduce uncooled IRFPA production cost.

NETD in the range of $30 \mathrm{mK}(\mathrm{f} / 1,300 \mathrm{~K}, 60 \mathrm{~Hz})$ as well as operability higher than $99.99 \%$, are routinely achieved with amorphous silicon technology.
\end{abstract}

Keywords: uncooled microbolometer, amorphous silicon, IRFPA.

\section{INTRODUCTION}

From $45 \mu \mathrm{m}$ pixel pitch $(320 \times 240)$ in 2002, $35 \mu \mathrm{m}$ pixel pitch $(160 \times 120$ and $384 \times 288)$ in $2003,25 \mu \mathrm{m}$ pixel pitch $(640 \times 480,384 \times 288$ and $160 \times 120)$ in 2005 and $17 \mu \mathrm{m}(1024 \times 768)$ in 2008, ULIS has demonstrated the capability to produce IR uncooled detectors with high operability and high performance. These detectors are available for various applications. Their simple operating conditions are similar to those of digital CMOS Active Pixel Sensors (APS) for visible applications. They have already shown their potential to fulfill many commercial (thermography, fire-fighter, automotive, medical ...) and military (thermal weapon sight, DVE) applications.

As the field of applications expands, the market needs are oriented not only towards higher performance but mainly on reduced size and reduced cost. Since 2005, ULIS has made a significant breakthrough in designing the first uncooled 1/16 TV format $25 \mu \mathrm{m}$ pixel pitch TEC-less detector designed for low-end applications $\left[{ }^{1}\right]$. This paper describes the main parameters (detector technology and package type) which drive the uncooled detector development.

\section{GENERAL CHARACTERISTICS}

\subsection{Detector technology}

Amorphous silicon material is well known for many applications and its use for uncooled infrared detector production benefits from a simple technology easier to master than other technologies making use of different material $\left[{ }^{2}\right]\left[{ }^{3}\right]\left[^{4}\right]$. The advantage of amorphous silicon is to be easily integrated onto silicon substrate at temperature compatible with CMOS readout integrated circuit using well mastered deposition and etching technologies. By pushing the design rules closer to the limit, the amorphous silicon technology enables the manufacturing of detector arrays based on $17 \mu \mathrm{m}$ pixel size and promises drastic 
improvement in system size and cost reduction while keeping the thermal time constant as low as $<10$ ms.

\subsection{Readout circuit}

The readout integrated circuit design (figure 1) includes a serial programmable interface, which allows the operation of the device to be optimized for a wide range of conditions and provides a large degree of flexibility. The array operates in non-interlaced mode. Integration of row $\mathrm{n}$ is controlled by a specific clock, and data is sampled and held, and read out during integration of row $n+1$. Moreover, the ROIC has an onchip programmable gain, which allows, with integration time and active microbolometer bias, optimization of performance over a wide range of operating conditions. These settings can also be used to optimize or to manage the trade off between scene dynamic range and sensitivity, according to different applications.

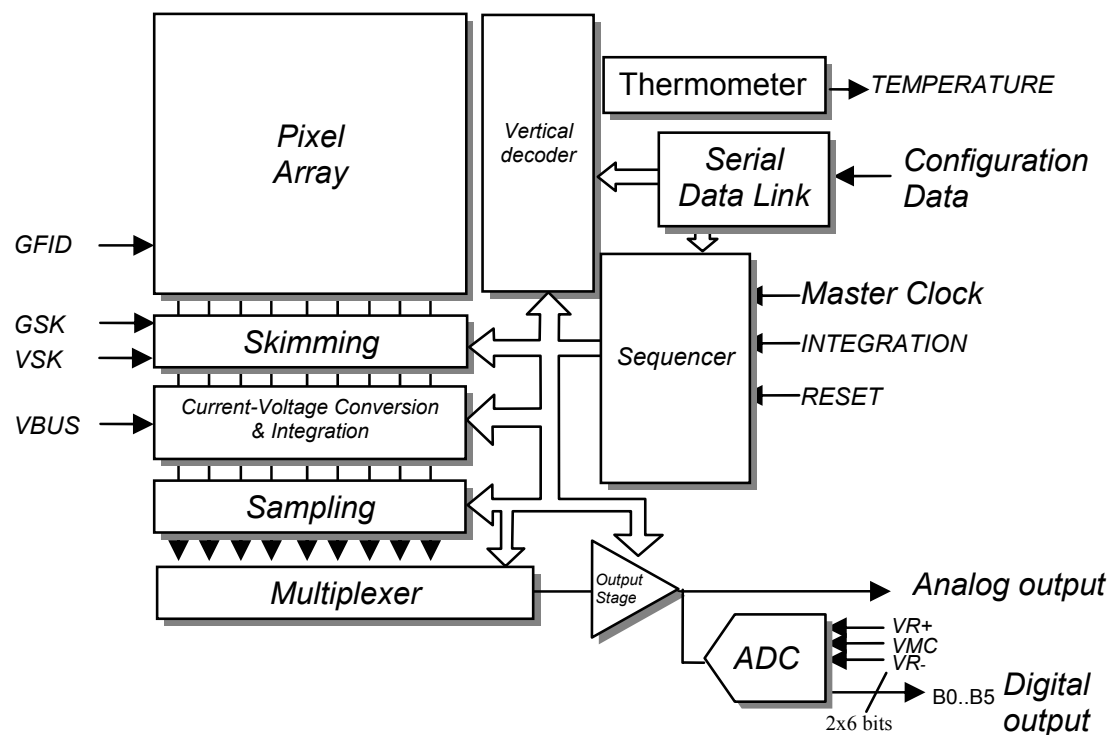

Figure 1: ROIC scheme

The last improvement consists of the implementation of a 13 bits on-chip analog to digital converter $(A D C)$. This $A D C$ has 12 efficient number of bits (ENOB) multiplexed in $2 \times 6$ bits in order to limit the package I/O number and hence package size. The user could use either the analog or the digital output at its own convenience. The ADC design is based on a pipeline architecture where three specific biases (VR+, VR- and VMC) allow driving the output dynamic range and then optimizing the quantization [ ${ }^{5}$ ].

\section{SENSOR DESIGNS}

Uncooled IRFPA market needs could be divided into two categories:

- High performance and hard environment applications,

- High volume applications.

We have developed to answer these needs, two types of package: a metallic package with embedded thermo-electric cooler (TEC) and a ceramics package without TEC. This second configuration opens the possibility of a less expensive collective process to package the microbolometer chip under vacuum (see figure 2) at the advantage of a low power consumption with TEC-less sensor operation.

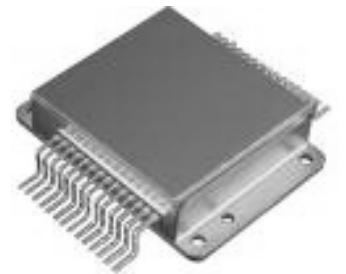

a

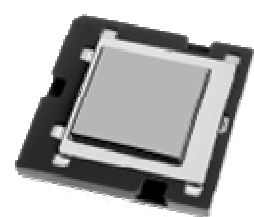

b

Figure 2: Metallic (a) and ceramics (b) packages developed for IRFPA 


\subsection{High performance sensors}

Typical NETD of high performance sensors is less than $30 \mathrm{mK}, \mathrm{f} / 1,60 \mathrm{~Hz}$ in $384 \times 288$ configuration (Figure 3) and is routinely achieved with $25 \mu \mathrm{m}$ pixel size sensor. The typical NETD Gaussian distribution shows a standard deviation less than $7 \mathrm{mk}$ which illustrates the high uniformity of amorphous silicon technology. As a consequence, such a detector does not need any additional on-chip non-uniformity compensation device.

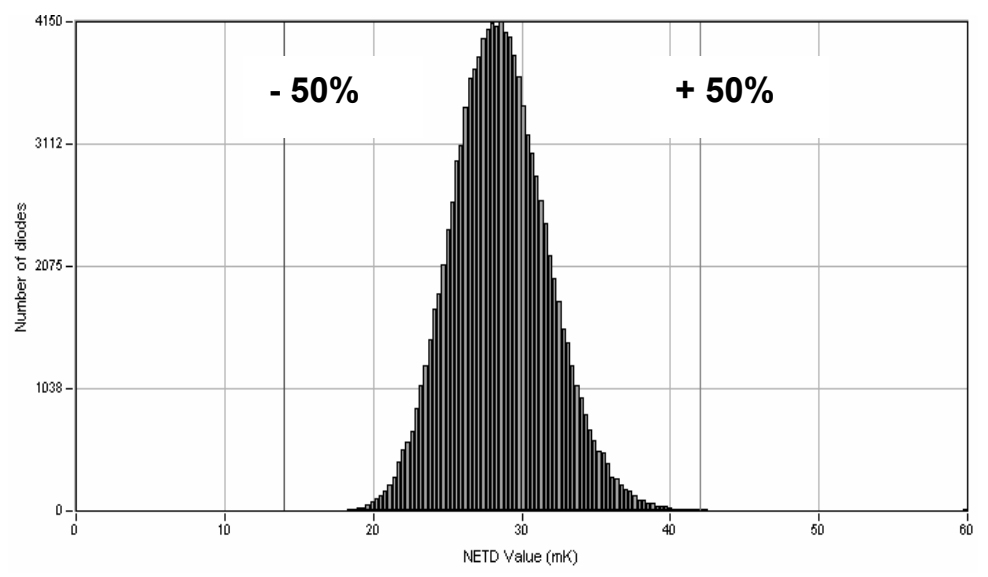

Figure 3: Typical NETD distribution for a typical array

These sensors are specifically designed to sustain harsh environment (Figure 2a) in terms of mechanical shocks, vibrations and thermal cycling which enables to use this kind of sensors for thermal weapons sight or other demanding applications.

Development of pixel size of $17 \mu \mathrm{m}$ enables to provide high definition IR image $\left[{ }^{6}\right]$ (see figure 4 ).

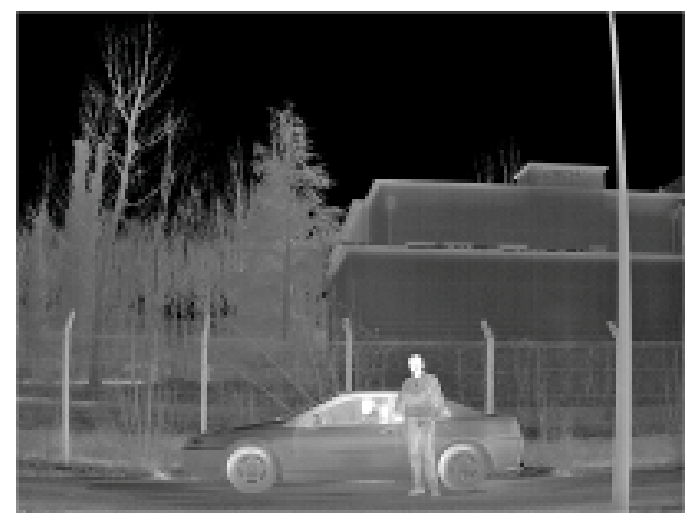

Figure 4: High definition IR 1024 x 768 image with $17 \mu \mathrm{m}$ pixel size

Taking profit from amorphous silicon properties, $1024 \times 768 / 17 \mu \mathrm{m}$ devices show NETD better than $50 \mathrm{mK}$.

\subsection{High volume sensors}

For high volume applications, the $384 \times 288 / 25 \mu \mathrm{m}$ focal plane arrays are integrated under vacuum in specifically designed ceramic packages (figure $2 \mathrm{~b}$ ). This extremely thin package is sealed under vacuum without using the traditional pinch-off tube, leading to a more compact package with less impact on the electronic board design. The distance between the FPA and the external window surface enables the use of short back working-distance optics. Package design is very important for vacuum life span as well as manufacturability. In line with our most up to date knowledge, the vacuum life span exceeds a 15 year storage life time at room temperature. The package is compliant with the RoHs regulation which has had to be applied since mid 2006. Although the detector is designed for TEC-less operation, a heater has been integrated for more demanding applications.

All electrical and electro-optical tests are defined and carried out with f/1 optical aperture, a background temperature of $293 \mathrm{~K}, 313 \mathrm{~K}$ FPA temperature and $60 \mathrm{~Hz}$ frame rate. The circuit is operated in digital mode. The mean responsivity value is $5.3 \mathrm{mV} / \mathrm{K}$ with $\sigma / \mathrm{m}$ as low as $1.1 \%$. This very high responsivity 
uniformity is the result of the amorphous silicon technology developed for uncooled infrared detector production.

Typical operability is better than $99.9 \%$. The mean NETD is equal to $85 \mathrm{mK}$ with a standard deviation equal to $10 \mathrm{mK}$. To characterize the image quality, it's important to measure the Residual Fixed Pattern Noise (RFPN) after two point correction. Figure 4 shows an image covering a scene temperature range of $4^{\circ} \mathrm{C}$ (this detector comprises only 3 defective pixels). Correction points are $20^{\circ} \mathrm{C}$ and $50^{\circ} \mathrm{C}$. The FPN map was taken in front of a blackbody at $35^{\circ} \mathrm{C}$. The RFPN mean value is in these conditions equal to $361 \mu \mathrm{V}$ which is approximately $70 \%$ of the RMS temporal noise.

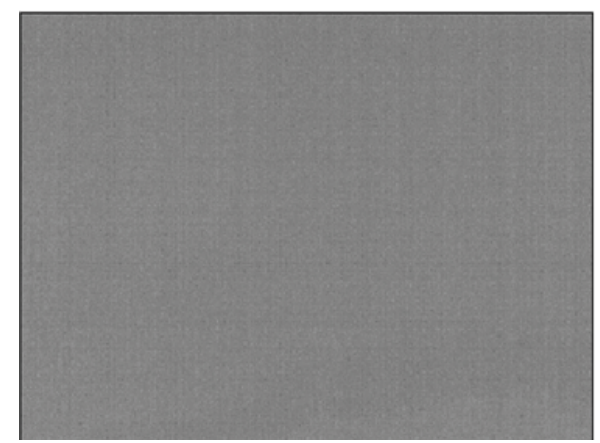

Figure 5: Residual fixed pattern noise $\left(4^{\circ} \mathrm{C}\right.$ range $)$ on $384 \times 288$ pixels format

Besides, a very advanced packaging technique is currently under development $\left[^{7}\right]$. This unique patented technology leads to an ultimate sensor cost reduction by using a pixel level package instead of wafer level package. It means that no additional technological steps are necessary to integrate the FPA chip under vacuum as the microbolometer process comprises itself the vacuum integration at the pixel level. Figure 6 shows a first technology demonstrator. Each cell contains a $25 \mu \mathrm{m} \times 25 \mu \mathrm{m}$ microbolometer pixel.

This new technology paves the way to very high volume sensor applications (i.e. automotive application).

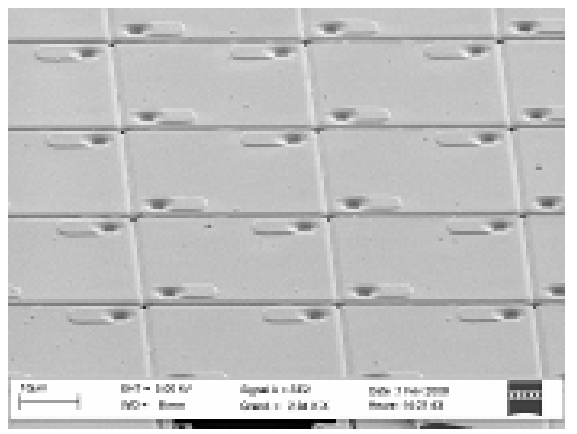

Figure 6: Very advanced monolithic on-chip vacuum packaging

\section{CONCLUSION}

A significant breakthrough has been achieved in the development of amorphous silicon $25 \mu \mathrm{m}$ and now $17 \mu \mathrm{m}$ pixel-pitch technologies to address high end and high volume applications. Detectors show performance comparable to that of $35 \mu \mathrm{m}$ arrays with outstanding pixel operability and uncorrected responsivity non-uniformity. A complete summary of the electro-optical performances has been reviewed, showing a top performance better than $30 \mathrm{mK}, \mathrm{F} / 1,60 \mathrm{~Hz}$. The manufacturing aspect shows that amorphous silicon technology remains ahead mainly due to its remarkable simplicity, uniformity and repeatability, facilitating the detector mass production needed by the market. Such a sensor with state of the art performances and reliable proven package will fit most of applications.

A read out integrated circuit structure with high level functions like serial link and 13 bits on-chip ADC make an easier operation. A thin ceramic package has been developed enabling one the possibility to build compact hand-held or helmet-mounted cameras for high volume applications. The TEC-less mode operability is completely demonstrated on a wide ambient temperature range. This feature opens the way to reduced power consumption systems needed for man-portable applications.

However, further packaging improvement is under progress to address very high volume application like automotive or radiometry. 


\section{Acknowledgments}

The authors would like to thank the uncooled detectors Teams of LETI LIR and ULIS who develop and produce uncooled infrared microbolometer detectors. We also thank the Sofradir staff for their contribution and their support.

\section{References}

1 “Compact uncooled amorphous silicon 160x120 IRFPA with $25 \mu \mathrm{m}$ pixel-pitch for large volume applications”, J.L.Tissot, O. Legras, C. Trouilleau, B. Dupont, B. Fieque, S. Tinnes, C. Minassian, J. Yon, European Security and Defense, Proc. of SPIE Vol. 6737, (2007).

2 "35 $\mu \mathrm{m}$ pitch at ULIS, a breakthrough", C. Trouilleau, A. Crastes, O. Legras, J.L. Tissot, J.P. Chatard, Infrared Technology and Applications XXXI, Proc. of SPIE Vol. 5783, (2005), pp 578-585

3 "320x240 microbolometer uncooled IRFPA development", J.L. Tissot et al, Infrared technology and Applications XXVI, proc. of SPIE Vol. 4130, (2000), pp. 473-479

4 "Microbolometer development and production at Indigo Systems", W. Terre et al, Infrared Technology and Applications XXIV, proc. of SPIE Vol. 5074 (2003), pp. 518-526

5 "Design trade-offs in ADC architectures dedicated to uncooled infrared focal plane arrays", P. Robert, B. Dupont, D. Pochic, , Infrared technology and Applications XXXIV, proc. of SPIE Vol. 6940, (2008).

6 "Uncooled amorphous silicon XGA IRFPA with $17 \mu \mathrm{m}$ pixel-pitch for High End applications", B. Fièque, P. Robert, C. Minassian, M Vilain, J.L. Tissot, A. Crastes, O. Legras, JJ Yon, Infrared technology and Applications XXXIV, proc. of SPIE Vol. 6940, (2008)

7 "Innovative on-chip packaging applied to uncooled IRFPA", G. Dumont et al, Infrared technology and Applications XXXIV, proc. of SPIE Vol. 6940, (2008). 